\title{
Subclinical sacroiliitis detected by abdominopelvic computed tomography in Korean patients with Crohn's disease
}

\author{
You-Jung $\mathrm{Ha}^{1,2,}{ }^{,}$, Hyo Jin Kim ${ }^{3,}$, Eugene Lee ${ }^{4}$, Ji Hye Park ${ }^{5}$, Young Soo Park ${ }^{5}$, Yun Jong Lee ${ }^{1,2}$,
} Yusuhn Kang ${ }^{4}$, and Hyuk Yoon ${ }^{5}$

\begin{abstract}
${ }^{1}$ Division of Rheumatology, Department of Internal Medicine, Seoul National University Bundang Hospital, Seongnam; ${ }^{2}$ Department of Internal Medicine, Seoul National University College of Medicine, Seoul; ${ }^{3}$ Department of Radiology, Seoul Metropolitan Government Seoul National University Boramae Medical Center, Seoul; Departments of ${ }^{4}$ Radiology and ${ }^{5}$ Internal Medicine, Seoul National University Bundang Hospital, Seongnam, Korea
\end{abstract}

\section{Correspondence to Hyuk Yoon, M.D. \\ Department of Internal Medicine, Seoul National University Bundang Hospital, 82 Gumi-ro 173beon-gil, Bundang- gu, Seongnam 13620, Korea Tel: +82-31-787-7009 \\ Fax: +82-31-787-4051 E-mail: bodnsoul@hanmail.net https://orcid.org/0000-0002- 2657-0349}

\section{Yusuhn Kang, M.D.}

Department of Radiology, Seoul National University Bundang Hospital, 82 Gumi-ro 173beongil, Bundang-gu, Seongnam 13620 , Korea

Tel: $+82-31-787-7606$

Fax: +82-31-787-4011

E-mail: yskango114@gmail.com https://orcid.org/0000-0003$1838-2564$

*'These authors contributed equally to this work.

Background/Aims: Sacroiliitis is a frequent extraintestinal manifestation of inflammatory bowel diseases (IBDs). This study aimed to assess the prevalence of sacroiliitis using a validated screening tool based on abdominopelvic computed tomography (APCT) in Korean patients with Crohn's disease (CD) and examine potential associations between clinical characteristics and sacroiliitis.

Methods: One hundred five patients with CD undergoing APCT for any indication at an IBD clinic were matched 1:1 for age and sex with 105 controls without underlying chronic illnesses. Using a validated APCT screening tool that defines sacroiliitis as either ankylosis or a total erosion score (TES) $\geq 3$, all computed tomography scans were assessed by two independent, blinded radiologists. We compared the prevalence of sacroiliitis between CD patients and controls and clinical characteristics between CD patients with and without sacroiliitis.

Results: The prevalence of sacroiliitis was significantly higher in CD patients than in controls ( $13.3 \%$ vs. $4.8 \%, p=0.030$ ). All subjects with sacroiliitis had a TES $\geq 3$, but no ankylosis. The assessment of sacroiliitis in APCT showed excellent interreader reliability (Cohen's kappa $=0.933$ for presence of sacroiliitis). Sacroiliitis in CD patients was bilateral and asymptomatic. There were no significant associations between sacroiliitis and any demographic data or clinical characteristics in these patients.

Conclusions: The prevalence of APCT-detected sacroiliitis in CD patients was higher than that in controls, but the condition was asymptomatic. The clinical significance of asymptomatic sacroiliitis in Korean CD patients remains unclear.

Keywords: Sacroiliitis; Sacroiliac joint; Tomography, spiral computed; Crohn disease

Received : May 4, 2020, Revised : July 1, 2020, Accepted : July 3, 2020

\section{INTRODUCTION}

Inflammatory bowel disease (IBD) comprises ulcerative colitis (UC) and Crohn's disease $(\mathrm{CD})$ and is a chronic, relapsing inflammatory disease of the gastrointestinal tract of unknown cause $[1,2]$. Almost half of the patients with IBD 
exhibit extraintestinal manifestations (EIMs) affecting the skin, joints, eyes, hepatobiliary system, or other body parts in their lifetime [3-5]. Musculoskeletal involvement is the most common EIM in IBD, ranging from articular and periarticular features to muscle and bone involvement [6]. The reported prevalence of arthropathy in IBD varies from $6 \%$ to $46 \%$ of patients, depending on the population and definition of joint involvement. Because joint symptoms can adversely affect the future health-related quality of life in IBD patients, early detection and treatment could aid in the improvement of the overall prognosis [7].

Arthritis associated with IBD, also called enteropathic arthritis, belongs to the spondyloarthritis (SpA) group of interrelated, but phenotypically distinct disorders, along with ankylosing spondylitis (AS), psoriatic arthritis, and a subgroup of juvenile idiopathic arthritis [8,9]. According to the main joints involved, SpA can be divided into a peripheral type and an axial type (including sacroiliitis with or without spondylitis) that can occur either independently or in combination. The association between IBD and SpA has been well established. In a large population-based IBD cohort, AS occurred in 3.7\% and SpA in $22 \%$ of patients after the IBD diagnosis [10]. On the other hand, IBD can develop in approximately $5 \%$ to $10 \%$ of patients diagnosed with AS [11,12]. The reported prevalence of radiographic sacroiliitis (asymptomatic or symptomatic) in patients with IBD ranges widely from $2 \%$ to $46 \%$ of patients with IBD [13].

In clinical practice, considerable numbers of patients with IBD undergo abdominopelvic computed tomography (APCT) scans for a variety of clinical purposes. Recently, a Canadian study group [14] developed and suggested a screening tool for identifying sacroiliitis on APCT images that can be used by both rheumatologists and radiologist. This tool is a firstly validated method to classify sacroiliitis using existing computed tomography (CT) scans. Subsequently, two Canadian IBD cohort studies $[15,16]$ described the prevalence and clinical association of sacroiliitis with IBD using the tool. Because sacroiliitis is often asymptomatic and may be underdiagnosed in IBD patients [17], the application of this tool on APCT images can assist in the identification of subclinical sacroiliitis early during the course of disease, without the need for additional evaluations. In Korean patients with IBD, data on the exact prevalence and clin- ical characteristics of enteropathic arthritis is scarce so far. A study by Suh et al. [18] two decades ago reported a low prevalence of axial involvement with $6.2 \%$ of patients showing sacroiliitis on plain radiographs. CT shows a higher sensitivity and lower interobserver variability than conventional radiography in the detection of sacroiliitis [19].

The aim of this study was to assess the prevalence of sacroiliitis by applying the validated APCT tool in a cohort of Korean CD patients and matched controls and to investigate the clinical characteristics of the $\mathrm{CD}$ patients with sacroiliitis.

\section{METHODS}

\section{Study population}

From April 2017, we initiated the set-up of the IBD cohort and enrolled all patients who visited the IBD clinic at Seoul National University Bundang Hospital (SNUBH, Seongnam, Korea). For the present study, individuals with $\mathrm{CD}$ who underwent APCT scan from June 2011 to September 2018 $(\mathrm{n}=114)$ were identified from this cohort. Patients with UC were excluded because of the small number of patients who underwent APCT scans. We further excluded APCT scans that were regarded as unsuitable for the analysis with the screening tool by the radiologists. The selection of patients for this study is shown in Fig. 1. Among 114 APCT scans in CD patients, scans suggesting osteopetrosis, osteitis condensans ilii, and diffuse idiopathic skeletal hyperostosis (each $n=1$ ) and those with contiguous cut thickness $\geq 5 \mathrm{~mm}(\mathrm{n}=6)$ were excluded. Finally, a total of 105 patients were enrolled and analyzed as the $\mathrm{CD}$ patient group.

A total of 105 sex- and age-matched control patients were randomly selected from the SNUBH medical imaging database among subjects who visited the emergency room and underwent an APCT for any indication from January 2016 to December 2018 and were described as previously healthy. The electronic medical charts of each control patient before and after the emergency room visit were reviewed, and patients with IBD, autoimmune diseases (e.g., inflammatory arthritides such as rheumatoid arthritis, spondyloarthropathy, and psoriatic arthritis or connective tissue diseases), malignancies, chronic illnesses (hypertension, diabetes, and 


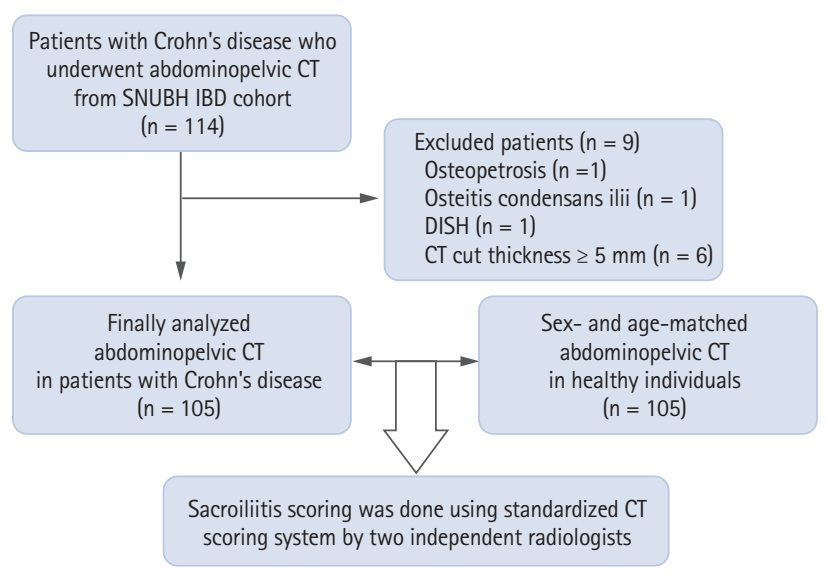

Figure 1. Participant selection for this study on sacroiliitis in patients with Crohn's disease $(n=114)$. CT, computed tomography; SNUBH, Seoul National University Bundang Hospital; IBD, inflammatory bowel diseases; DISH, diffuse idiopathic skeletal hyperostosis.

cardiovascular diseases) and chronic back pain were excluded. The indications for the CT scan were recorded for all controls.

The Institutional Review Board of the SNUBH approved the study protocol (IRB No. B-1908/561-103) and waived the need for informed patient consent due to the retrospective study design.

\section{Data collection}

Clinical information at the time of CT scanning was collected for all CD patients and included demographic variables, disease duration, EIMs, and the use of medications, such as corticosteroids, immunomodulators, and biologics. All patients were classified using the Montreal classification as follows [20]; L1, disease limited to the terminal ileum; L2, disease location at any position between the cecum and rectum; L3, involvement of the terminal ileum and the colon; and L4, any disease location proximal to the terminal ileum; $\mathrm{B} 1$, non-stricturing, non-penetrating; $\mathrm{B}_{2}$, stricturing; and $\mathrm{B}_{3}$, penetrating; A1, age below 16 years; A2, age between 17 and 40 years; and $A_{3}$, age above 40 years. Laboratory variables within one week of the APCT were recorded and included the white blood cell count, hemoglobin, erythrocyte sedimentation rate (ESR), C-reactive protein (CRP), and fecal calprotectin. The presence of immunoglobulin $\mathrm{G}(\mathrm{IgG})$ or IgA anti-Saccharomyces cerevisiae antibodies (ASCA) and anti-neutrophil cytoplasmic antibodies (ANCA) identified with indirect immunofluorescence, was also recorded. Some of the patients completed a questionnaire aimed at detecting arthritis in IBD patients not previously diagnosed with $\operatorname{SpA}[21]$.

\section{Assessment of sacroiliitis}

Sacroiliitis was assessed using the previously reported APCT scoring system [14]. Briefly, the sacroiliac joints were divided into four segments (left/right and iliac/ sacral side). An erosion score was allocated to each segment, by identifying the image slice with the greatest number of erosions. The total erosion score (TES) was calculated by adding the erosion scores of the four segments. Ankylosis was defined as contiguous bone marrow between the ilium and sacrum within the cartilage compartment, exceeding $1 \mathrm{~cm}$ in width. The presence of sclerosis was also recorded, although it was not incorporated into the element of judgement for sacroiliitis. The presence of either ankylosis or a TES $\geq 3$ was classified as sacroiliitis.

All CT images were independently reviewed by two experienced musculoskeletal radiologists (Y.K. and H.K.) blinded to the patients' clinical information. Arbitration of discordant readings was performed by a third radiologist (E.L.).

\section{Statistical analysis}

Continuous variables were expressed as mean \pm standard deviation, and categorical variables as numbers and percentage (\%). Continuous variables were compared using the Student's $t$ test (or Mann-Whitney test, if appropriate), and categorical variables were compared by the chisquare test or Fisher's exact test. Interreader reliability was assessed using a kappa for nominal variables and an intraclass correlation coefficient (ICC) for continuous variables. All analyses were performed using STATA SE version 15.0 (StataCorp LLC, College Station, TX, USA). A $p<0.05$ was considered statistically significant.

\section{RESULTS}

\section{Characteristics of the study population and control}

The demographic data of the CD patients and controls are shown in Table 1 . The mean age of the study population was $29.3 \pm 10.6$ years, and $77 \%$ of patients were 
Table 1. Baseline demographics and prevalence of sacroiliitis and its features in Korean patients with Crohn's disease (n = 105) and control group $(n=105)$

\begin{tabular}{|c|c|c|c|}
\hline Variable & Crohn's disease $(\mathrm{n}=105)$ & Control $(\mathrm{n}=105)$ & $p$ value \\
\hline Age, yr & $29.3 \pm 10.6$ & $29 \cdot 3 \pm 10.6$ & NA \\
\hline Male sex & $81(77 \cdot 1)$ & $81(77 \cdot 1)$ & NA \\
\hline Presence of sacroiliitis & $14(13 \cdot 3)$ & $5(4.8)$ & $0.030^{\mathrm{a}}$ \\
\hline Ankylosis & o & 0 & NA \\
\hline Total erosion score & $0.89 \pm 0.13$ & $0.55 \pm 0.08$ & $0.032^{\mathrm{a}}$ \\
\hline Total erosion score $\geq 3$ & $14(13 \cdot 3)$ & $5(4.8)$ & $0.030^{\mathrm{a}}$ \\
\hline Presence of any site sclerosis & $2(1.9)$ & $2(1.9)$ & 1.000 \\
\hline Bilateral & $14 / 14(100)$ & $4 / 5(80)$ & 0.086 \\
\hline
\end{tabular}

Values are presented as mean \pm standard deviation or number (\%).

NA, not applicable.

${ }^{a}$ Significant at $5 \%$ level of significance.

male. The reasons for performing an APCT scan in the control patients were acute abdominal pain (74.3\%), fever of unknown origin (14.3\%), flank pain (4.8\%), minor trauma after traffic accidents (2.8\%), and hematochezia or diarrhea (2.8\%). None of the control patients had been diagnosed with SpA prior to the APCT based on their medical record review.

\section{Prevalence of sacroiliitis in CD patients and controls}

Fig. 2 displays a representative image of erosion among $\mathrm{CD}$ patients. According to the CT scoring tool, sacroiliitis was found in $13.3 \%$ of the CD patients and $4.8 \%$

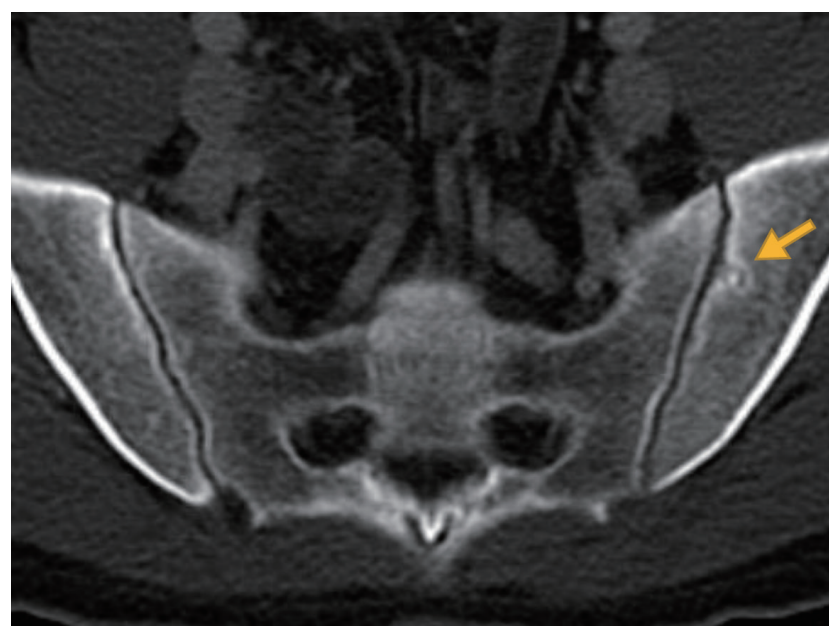

Figure 2. Axial view of abdominopelvic computed tomography scan showed an erosion on the left iliac side of the sacroiliac joint (yellow arrow). of the controls, which was a statistically significant difference $(p=0.03$ ) (Table 1$)$. All subjects with sacroiliitis met the criterion of a TES $\geq 3$, but none had ankylosis. The distribution of TES in the two groups is shown in Fig. 3. The TES of all controls with sacroiliitis was three points. In CD patients with sacroiliitis, the majority had a TES of three points, and the maximum TES was six points. None of these patients had been referred to a rheumatologist. The abdominal radiologists who originally read the CT scans, did not comment on skeletal changes in all patients with sacroiliitis. Involvement of sacroiliac joint was bilateral in all CD patients and $80 \%$

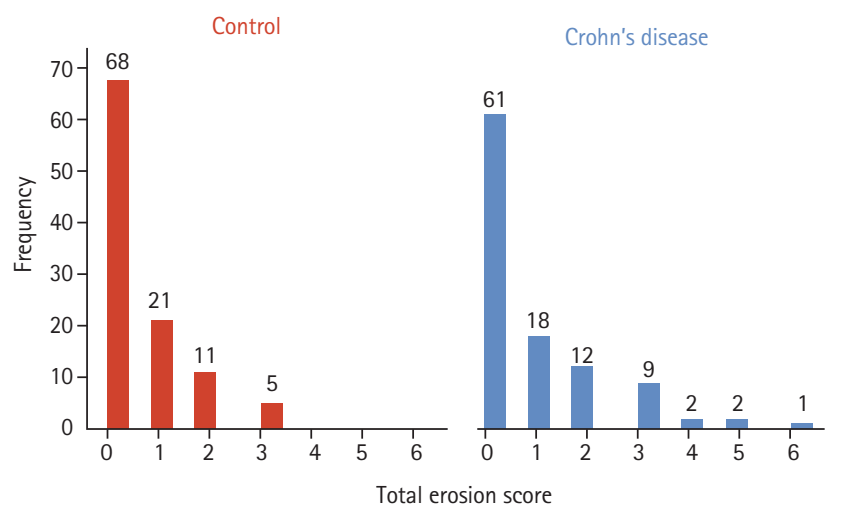

Figure 3. Total erosion score distributions in patients with Crohn's disease $(n=105)$ and controls $(n=105)$. 
Table 2. Interreader reliability for the computed tomography examination of the sacroiliac joints in all subjects $(n=210)$

\begin{tabular}{|c|c|}
\hline Variable & Interreader reliability \\
\hline Ankylosis (к) & 0.995 \\
\hline Presence of erosion ( $(\kappa)$ & 0.938 \\
\hline Total erosion score (ICC) & 0.891 \\
\hline Sclerosis $(\kappa)$ & 0.981 \\
\hline Presence of sacroiliitis ( $(\kappa)$ & 0.933 \\
\hline
\end{tabular}

ICC, intraclass correlation coefficient.

of individuals in the control group. The interreader reliability between the radiologists in their assessment of the sacroiliac joint is shown in Table 2, showing excellent interreader agreement (Cohen's kappa $=0.933$ for presence of sacroiliitis).

\section{Associations of sacroiliitis with clinical features of CD}

We compared the clinical and laboratory characteristics between CD patients with and without sacroiliitis (Table 3). There were no differences in the demographic data, disease duration, location, behavior, prevalence of EIMs, and history of hospitalizations or emergency room visits. Immunomodulators were less frequently used in CD patients with sacroiliitis, but this difference was not statistically significant $(p=0.057)$. Neither the laboratory markers of inflammation, such as ESR, CRP, and fecal calprotectin nor the autoantibodies ASCA and ANCA were significantly associated with the presence of sacroiliitis. Crohn's disease activity index (CDAI) between two groups did not differ, although available in small portion of patients (27.6\%). Among the $14 \mathrm{CD}$ patients with sacroiliitis, 11 patients completed the self-administrated questionnaire for detecting arthritis in IBD patients (Korean version of DETection of Arthritis in Inflammatory boweL diseases [DETAIL]) [21]. All of them denied inflammatory back pain, so they were considered having subclinical sacroiliitis.

\section{DISCUSSION}

Our data show that a substantial proportion of patients (13.6\%) with established CD have undetected subclinical sacroiliitis. Sacroiliitis in CD patients was bilateral and asymptomatic. The prevalence of sacroiliitis was high- er in CD patients than in healthy individuals. However, the clinical significance of APCT-detected sacroiliitis remains uncertain in the Korean $\mathrm{CD}$ population. It has been reported that patients with radiographic findings of bilateral sacroiliitis are more likely to progress to AS and occult radiographic alterations in the sacroiliac joints usually precede clinical symptoms of inflammatory back pain $[22,23]$. Hence, the application of this screening tool on acquired APCT images could aid in the early identification of IBD patients at high risk for developing SpA and enable early treatment.

Musculoskeletal problems, including joint complaints, are the most common EIM in IBD patients [4]. The presence of SpA can negatively impact on health-related quality of life and cause an additional burden in IBD patients $[6,7]$. It has been found that axial involvement is seen in up to $45 \%$ of patients with IBD depending on the definition of axial involvement, imaging modalities used and patient population. Previous studies using plain X-ray have reported a prevalence of sacroiliitis ranging from $9.2 \%$ to $24 \%$ in IBD patients $[24,25]$. In particular, asymptomatic sacroiliitis has been reported to be common in CD patients [26]. In a Korean study that assessed sacroiliitis based on the New York grading system using CT, sacroiliitis was identified in $16.6 \%$ of IBD patients [27]. In the Canadian cohort study that first developed and validated the screening tool used in our study, sacroiliitis was found in $17 \%$ of CD patients. In a recent study analyzing the sacroiliac joint with magnetic resonance colonography and enterography, inflammatory sacroiliitis was detected in $16.7 \%$ of IBD patients [28]. Therefore, our result of $13.6 \%$ is similar to the previously reported prevalence of sacroiliitis using magnetic resonance imaging (MRI) or CT in IBD patients.

For detecting sacroiliitis, plain radiographs have been used as the first-line imaging tool. However, the irregular outline and obliquity of the sacroiliac joints make it difficult to determine the grading of sacroiliitis on radiographs [29]. This limitation has been surmounted by more sensitive imaging modalities such as CT and MRI, which show excellent ability in detecting erosions, bone sclerosis and ankylosis [30]. CT is superior in detecting chronic structural changes, but inadequate in assessing active inflammatory lesions that suggest sacroiliitis. Consequently, MRI is the gold standard for diagnosing SpA and assessing disease severity and activity. How- 
Table 3. Comparison of the demographics and clinical characteristics according to the presence of sacroiliitis

\begin{tabular}{|c|c|c|c|c|}
\hline Variable & Total $(\mathrm{n}=105)$ & Sacroiliitis $(n=14)$ & No sacroiliitis $(\mathrm{n}=91)$ & $p$ value \\
\hline Age at diagnosis & $26.5 \pm 10.4$ & $26.1 \pm 13.5$ & $26.5 \pm 9.9$ & 0.892 \\
\hline Age, yr & & & & 0.396 \\
\hline$<17$ & $8(7.6)$ & $2(14.3)$ & $6(6.6)$ & \\
\hline$\geq 17$ and $<40$ & $88(85.7)$ & $10(71.4)$ & $78(85.7)$ & \\
\hline$\geq 40$ & $9(8.6)$ & $2(14 \cdot 3)$ & $7(7.7)$ & \\
\hline Male sex & $81(77.1)$ & $10(71.4)$ & $71(78.0)$ & 0.584 \\
\hline Family history of IBD & $6(5.7)$ & $2(14.3)$ & $4(4 \cdot 4)$ & 0.138 \\
\hline Smoking & & & & 0.665 \\
\hline Never-smoker & $66(62.9)$ & $10(71.4)$ & $56(61.5)$ & \\
\hline Ex-smoker & $14(13 \cdot 3)$ & $2(14.3)$ & $12(13.2)$ & \\
\hline Current-smoker & $25(23.8)$ & $2(14 \cdot 3)$ & $23(25 \cdot 3)$ & \\
\hline Disease duration, yr & $2.81 \pm 4.25$ & $1.73 \pm 2.60$ & $2.98 \pm 4.43$ & 0.308 \\
\hline Location & & & & 0.373 \\
\hline $\mathrm{L} 1$ & $27(25.7)$ & $2(14 \cdot 3)$ & $25(27.5)$ & \\
\hline L2 & $3(2.9)$ & $1(7.1)$ & $2(2.2)$ & \\
\hline $\mathrm{L}_{3}$ & $75(71.4)$ & $11(78.6)$ & $64(70.3)$ & \\
\hline $\mathrm{L}_{4}$ & $12(11.4)$ & o & $12(13.2)$ & 0.149 \\
\hline Behavior & & & & 0.180 \\
\hline $\mathrm{B} 1$ & $52(49.5)$ & $9(64 \cdot 3)$ & $43(47 \cdot 3)$ & \\
\hline $\mathrm{B}_{2}$ & $15(14.3)$ & $3(21.4)$ & $12(13.2)$ & \\
\hline $\mathrm{B}_{3}$ & $38(36.2)$ & $2(14 \cdot 3)$ & $36(39.6)$ & \\
\hline Perianal disease & $43(41.0)$ & $4(28.6)$ & $39(42.9)$ & 0.312 \\
\hline CDAI (available no.) & $172.2 \pm 25.7(29)$ & $136.5 \pm 124.5(2)$ & $174.8 \pm 26.7(27)$ & 0.713 \\
\hline \multicolumn{5}{|l|}{ Extraintestinal manifestation } \\
\hline Arthritis/arthralgia & $12(11.4)$ & $2(14 \cdot 3)$ & $10(11.0)$ & 0.718 \\
\hline Eye involvement & $4(3.8)$ & $1(7.1)$ & $3(3 \cdot 3)$ & 0.484 \\
\hline Skin involvement & $14(13 \cdot 3)$ & $1(7.1)$ & $13(14 \cdot 3)$ & 0.464 \\
\hline \multicolumn{5}{|l|}{ Operation history } \\
\hline Abdominal & $16(15.2)$ & o & $16(17.6)$ & 0.088 \\
\hline Perianal & $37(35.2)$ & $4(28.6)$ & $33(36.3)$ & 0.575 \\
\hline Hospitalization history & $77(73 \cdot 3)$ & $10(81.4)$ & $67(73.6)$ & 0.575 \\
\hline ER visit history & $60(57.1)$ & $7(50.0)$ & $53(58.2)$ & 0.562 \\
\hline \multicolumn{5}{|l|}{ Medications history } \\
\hline Corticosteroids & $6_{7}(63.8)$ & $9(64 \cdot 3)$ & $58(63.7)$ & 0.968 \\
\hline Immunomodulators & $62(59.1)$ & $5(35.7)$ & $57(62.6)$ & 0.057 \\
\hline Anti-TNF $\alpha$ & $27(25.7)$ & $3(21.4)$ & $24(26.4)$ & 0.693 \\
\hline \multicolumn{5}{|l|}{ Laboratory findings (observed no.) } \\
\hline White blood cell count, $/ \mathrm{mm}^{3}$ & $8,069 \pm 359(91)$ & $8,228 \pm 879(11)$ & $8,047 \pm 392(80)$ & 0.870 \\
\hline Hemoglobin, mg/dL & $13.3 \pm 0.2(91)$ & $12.8 \pm 0.8(11)$ & $13.3 \pm 0.2(80)$ & 0.435 \\
\hline $\mathrm{ESR}, \mathrm{mm} / \mathrm{hr}$ & $27.1 \pm 3.8(82)$ & $27.7 \pm 12.6(10)$ & $27.0 \pm 4.0(72)$ & 0.952 \\
\hline
\end{tabular}


Table 3. Continued

\begin{tabular}{|c|c|c|c|c|}
\hline Variable & Total $(\mathrm{n}=105)$ & Sacroiliitis $(\mathrm{n}=14)$ & No sacroiliitis $(n=91)$ & $p$ value \\
\hline C-reactive protein, mg/dL & $2.8 \pm 0.5(88)$ & $2.2 \pm 0.9(11)$ & $2.9 \pm 0.6(77)$ & 0.671 \\
\hline Fecal calprotectin, mg/kg & $834.1 \pm 109.8(42)$ & $421.4 \pm 264.1(3)$ & $865.9 \pm 115.5(39)$ & 0.303 \\
\hline ASCA IgG & $29.1 \pm 2.1(98)$ & $24.0 \pm 4.4(14)$ & $29.9 \pm 2.3(84)$ & 0.328 \\
\hline Positive for ASCA IgG & $48(49.0)$ & $6(42.9)$ & $42(50.0)$ & 0.621 \\
\hline ASCA IgA & $17.8 \pm 1.5(73)$ & $23.9 \pm 6.2(11)$ & $16.7 \pm 1.4(62)$ & 0.093 \\
\hline Positive for ASCA IgA & $17(17.4)$ & $4(28.6)$ & $13(15 \cdot 5)$ & 0.231 \\
\hline Positive for ANCA IIF & $1 / 32(3.0)$ & $0 / 5(0)$ & $1 / 27(3.6)$ & 0.184 \\
\hline
\end{tabular}

Values are presented as mean \pm standard deviation or number (\%).

IBD, inflammatory bowel disease; CDAI, Crohn's disease activity index; ER, emergency room; TNF, tumor necrosis factor; ESR, erythrocyte sedimentation rate; ASCA, anti-Saccharomyces cerevisiae antibody; ANCA, anti-neutrophilic cytoplasmic antibody; Ig, immunoglobulin; IIF, indirect immunofluorescence.

ever, because of its long examination time and limited reimbursement criteria in Korean National Health Insurance system, MRI has not been widely implemented in CD patients. In the clinical practice, APCT scans have been commonly performed at the first diagnosis or emergent situations of CD patients. As the screening tool used in our study can use preexisting APCTs in IBD patients to assess the sacroiliac joint, its application enables early and more frequent detection of axial joint involvement without additional costs.

According to the Assessment of SpondyloArthritis International Society (ASAS) classification criteria for axial and peripheral SpA, CD is an item of both criteria $[8,31]$. If patients with $\mathrm{CD}$ have chronic inflammatory back pain and sacroiliitis on imaging, although CT is not included in it, they can be classified as having axial SpA. Because an APCT in these patients is usually assessed by radiologists specialized in gastrointestinal imaging, the musculoskeletal parts are frequently ignored. Indeed, in all our patients with sacroiliitis, there were no comments on the structural changes of the sacroiliac joint in the original report. Although CT is not included as a tool for determining sacroiliitis in the ASAS criteria, a free look of the sacroiliac joint on previously performed APCT by either gastroenterologists or radiologists help to refer potential patients to the rheumatology department and establish the diagnosis of SpA earlier.

Regarding the control group, it would be ideal to use APCT scans performed for medical check-up in healthy individuals or in patients with irritable bowel syn- drome. However, when reviewing the imaging database of our center, it was difficult to find APCT scans performed at the same age as CD patients. Also, there are few other specific indications to perform APCT scans in young healthy individuals. So, we assessed patients who had underwent APCT scans for any indications at the emergency room in healthy subjects. To minimize the bias that may be caused by inclusion of patients with chronic or inflammatory diseases as control group, medical charts of each control patient were meticulously reviewed to ensure them healthy.

Interestingly, a larger than expected percentage of patients (4.8\%) in the control group showed radiographic changes suggestive of sacroiliitis. This finding is consistent with the finding of Chan et al. [15], who showed that $5.6 \%$ of patients in the control group had sacroiliitis on CT. The presence of symptoms suggestive of SpA could not be investigated in this study due to its retrospective design, but musculoskeletal complaints were not reported in the medical records of these controls. These patients may have had undiagnosed SpA or other conditions showing sacroiliitis-like changes, including hyperparathyroidism and repetitive shear-stress injuries [32], or may have represented an overdiagnosis of sacroiliitis. Another MRI study that investigated sacroiliitis in healthy subjects and individuals with known mechanical strain acting showed that one-third to one-quarter of them had positive findings for sacroiliitis according to the ASAS definition [33]. The clinical significance and interpretation of sacroiliitis on imaging studies in as- 
ymptomatic or healthy subjects is a problem deserving further research in the future.

So far, there have been few studies investigating the epidemiology and clinical characteristics of axial involvement as an EIM in Korean IBD patients. In a study using Korean National Health Insurance data [34], the prevalence of established AS in CD patients was $0.48 \%$ compared to that of $0.09 \%$ in healthy controls, resulting in an adjusted odds ratio of 4.672. A retrospective study by Suh et al. [18] found radiographic sacroiliitis in $6.2 \%$ of IBD patients, who were all negative for human leukocyte antigen $\mathrm{B} 27$. There has been only one previous study [27] that used CT to detect sacroiliitis in Korean patients with IBD. In that study, sacroiliitis was diagnosed by the presence of at least unilateral sacroiliitis grade 1 on CT according to the New York grading system [35], which scores sacroiliitis on plain radiograph of pelvis: o, normal; 1, suspicious; 2, localized sclerosis, erosion, and joint widening; 3 , diffuse sclerosis, erosion, and widening; and 4, ankylosis. However, it had not been validated for CT images and a study by Geijer et al. [36] has suggested that application of the modified New York criteria on CT is inappropriate. So, the determination of sacroiliitis may have relied on non-validated radiologists' assessments. The authors also evaluated the clinical implications of sacroiliitis in IBD patients, but there was no comparison with a control group.

This study failed to establish an association of sacroiliitis with specific demographic data or clinical characteristics of CD patients. There have been several reports on the clinical significance of sacroiliitis in IBD patients that showed conflicting results. In a Belgian study [37], the presence of sacroiliitis on plain radiographs showed no association with age, sex, and phenotypes of CD. Meanwhile, Kelly et al. [16] demonstrated that sacroiliitis based on CT screening in Canadian IBD patients was associated with male sex, known arthritis, pain as an IBD symptom, and CD showing a non-penetrating behavior. Another study [27] showed that CT-detected sacroiliitis in CD patients was significantly associated with a certain location of the disease (perianal and upper gastrointestinal tract, corresponding to L4), but not with demographic data or CD behavior. Recently, data from a large-scale Swiss IBD cohort [38] showed that CD patients with sacroiliitis/AS were older and had a higher prevalence of other EIMs than patients without sacroiliitis, but there were no differences in the clinical $\mathrm{CD}$ features. These differences might be attributable to the ethnicity, small sample size, IBD type, inclusion of AS, and the definition of sacroiliitis. Further studies in larger numbers of multi-national patients are needed to clarify the clinical significance of sacroiliitis in IBD patients.

This study has some limitations. Firstly, because of its retrospective nature of our study, information about SpA symptoms was not systematically collected and dependent on the medical chart review. However, 11 of the 14 patients with sacroiliitis completed the DETAIL questionnaire [21], which can detect symptoms suggesting $\mathrm{SpA}$, and the remaining three patients had no axial symptoms recorded in their medical file. Secondly, an assessment of the clinical disease activity of $\mathrm{CD}$, such as the CDAI [39], was only available in a small number of patients, so an analysis about the association between sacroiliitis and clinical activity of CD was limited. Finally, due to the retrospective nature of the study, the CT image were acquired with various CT scanners and the image quality varied. This may have affected the results of our study. However, CT images with slice thickness of $5 \mathrm{~mm}$ or greater and those with suboptimal image resolution or presence of artifact were excluded from our study. Despite these limitations, the strength of our study include use of a validated APCT screening tool in Korean CD patients for the first time. Also, comparisons between CD patients and controls were conducted and radiologic assessments were done by plural radiologists with high interreader reliability. Although the APCT scans were analyzed retrospectively, clinical information of CD patients was retrieved from the systematically collected data of a prospective IBD cohort.

In conclusion, we found that the CT prevalence of sacroiliitis in Korean patients with CD was higher than that in the healthy control. Sacroiliitis is often subclinical and remains undiagnosed in clinical practice. There was no association between sacroiliitis and any clinical characteristics of CD in this population. Further research is required to explore the clinical significance of asymptomatic sacroiliitis in $\mathrm{CD}$ patients. 


\section{KEY MESSAGE}

1. A significant number of patients with Crohn's disease (CD, 13.6\%) have undetected subclinical sacroiliitis on preexisting abdominopelvic computed tomography.

2. The prevalence of sacroiliitis in CD patients is higher than in healthy controls, whereas no significant associations between sacroiliitis and any clinical features of CD were found in our Korean CD population.

\section{Conflict of interest}

No potential conflict of interest relevant to this article was reported.

\section{REFERENCES}

1. Zhang YZ, Li YY. Inflammatory bowel disease: pathogenesis. World J Gastroenterol 2014;20:91-99.

2. Mizoguchi E, Low D, Ezaki Y, Okada T. Recent updates on the basic mechanisms and pathogenesis of inflammatory bowel diseases in experimental animal models. Intest Res 2020;18:151-167.

3. Ott C, Scholmerich J. Extraintestinal manifestations and complications in IBD. Nat Rev Gastroenterol Hepatol 2013;10:585-595.

4. Vavricka SR, Schoepfer A, Scharl M, Lakatos PL, Navarini A, Rogler G. Extraintestinal manifestations of inflammatory bowel disease. Inflamm Bowel Dis 2015;21:1982-1992.

5. Kim JM, Cheon JH. Pathogenesis and clinical perspectives of extraintestinal manifestations in inflammatory bowel diseases. Intest Res 2020;18:249-264.

6. Colia R, Corrado A, Cantatore FP. Rheumatologic and extraintestinal manifestations of inflammatory bowel diseases. Ann Med 2016;48:577-585.

7. Ossum AM, Palm O, Cvancarova M, et al. The impact of spondyloarthritis and joint symptoms on health-related quality of life and fatigue in IBD patients: results from a population-based inception cohort (20-year follow-up in the ibsen study). Inflamm Bowel Dis 2020;26:114-124.

8. Rudwaleit M, van der Heijde D, Landewe R, et al. The development of Assessment of SpondyloArthritis International Society classification criteria for axial spondyloar- thritis (part II): validation and final selection. Ann Rheum Dis 2009;68:777-783.

9. Rudwaleit M, van der Heijde D, Landewe R, et al. The Assessment of SpondyloArthritis International Society classification criteria for peripheral spondyloarthritis and for spondyloarthritis in general. Ann Rheum Dis 2011;70:2531.

10. Palm O, Moum B, Ongre A, Gran JT. Prevalence of ankylosing spondylitis and other spondyloarthropathies among patients with inflammatory bowel disease: a population study (the IBSEN study). J Rheumatol 2002;29:511515 .

11. De Vos M, Mielants H, Cuvelier C, Elewaut A, Veys E. Long-term evolution of gut inflammation in patients with spondyloarthropathy. Gastroenterology 1996;110:16961703.

12. Stolwijk C, van Tubergen A, Castillo-Ortiz JD, Boonen A. Prevalence of extra-articular manifestations in patients with ankylosing spondylitis: a systematic review and meta-analysis. Ann Rheum Dis 2015;74:65-73.

13. Salvarani C, Fries W. Clinical features and epidemiology of spondyloarthritides associated with inflammatory bowel disease. World J Gastroenterol 2009;15:2449-2455.

14. Chan J, Sari I, Salonen D, Inman RD, Haroon N. Development of a screening tool for the identification of sacroiliitis in computed tomography scans of the abdomen. J Rheumatol 2016;43:1687-1694.

15. Chan J, Sari I, Salonen D, Silverberg MS, Haroon N, Inman RD. Prevalence of sacroiliitis in inflammatory bowel disease using a standardized computed tomography scoring system. Arthritis Care Res (Hoboken) 2018;70:807-810.

16. Kelly OB, Li N, Smith M, Chan J, Inman RD, Silverberg MS. The prevalence and clinical associations of subclinical sacroiliitis in inflammatory bowel disease. Inflamm Bowel Dis 2019;25:1066-1071.

17. Sheth T, Pitchumoni CS, Das KM. Musculoskeletal manifestations in inflammatory bowel disease: a revisit in search of immunopathophysiological mechanisms. J Clin Gastroenterol 2014;48:308-317.

18. Suh CH, Lee CH, Lee J, et al. Arthritic manifestations of inflammatory bowel disease. J Korean Med Sci 1998;13:39-43.

19. Navallas M, Ares J, Beltran B, Lisbona MP, Maymo J, Solano A. Sacroiliitis associated with axial spondyloarthropathy: new concepts and latest trends. Radiographics 2013;33:933-956.

20. Satsangi J, Silverberg MS, Vermeire S, Colombel JF. The 
Montreal classification of inflammatory bowel disease: controversies, consensus, and implications. Gut 2006;55:749-753.

21. Di Carlo M, Luchetti MM, Benfaremo D, et al. The DETection of Arthritis in Inflammatory boweL diseases (DETAIL) questionnaire: development and preliminary testing of a new tool to screen patients with inflammatory bowel disease for the presence of spondyloarthritis. Clin Rheumatol 2018;37:1037-1044.

22. Braun J, Sieper J. The sacroiliac joint in the spondyloarthropathies. Curr Opin Rheumatol 1996;8:275-287.

23. Bandinelli F, Terenzi R, Giovannini L, et al. Occult radiological sacroiliac abnormalities in patients with inflammatory bowel disease who do not present signs or symptoms of axial spondylitis. Clin Exp Rheumatol 2014;32:949-952.

24. Lanna CC, Ferrari Mde L, Rocha SL, Nascimento E, de Carvalho MA, da Cunha AS. A cross-sectional study of 130 Brazilian patients with Crohn's disease and ulcerative colitis: analysis of articular and ophthalmologic manifestations. Clin Rheumatol 2008;27:503-509.

25. Queiro R, Maiz O, Intxausti J, et al. Subclinical sacroiliitis in inflammatory bowel disease: a clinical and follow-up study. Clin Rheumatol 2000;19:445-449.

26. Atzeni F, Ardizzone S, Bertani L, Antivalle M, Batticciotto A, Sarzi-Puttini P. Combined therapeutic approach: inflammatory bowel diseases and peripheral or axial arthritis. World J Gastroenterol 2009;15:2469-2471.

27. Hwangbo Y, Kim HJ, Park JS, et al. Sacroiliitis is common in Crohn's disease patients with perianal or upper gastrointestinal involvement. Gut Liver 2010;4:338-344.

28. Leclerc-Jacob S, Lux G, Rat AC, et al. The prevalence of inflammatory sacroiliitis assessed on magnetic resonance imaging of inflammatory bowel disease: a retrospective study performed on 186 patients. Aliment Pharmacol Ther 2014;39:957-962.

29. Tsoi C, Griffith JF, Lee RKL, Wong PCH, Tam LS. Imaging of sacroiliitis: current status, limitations and pitfalls. Quant Imaging Med Surg 2019;9:318-335.
30. Baraliakos $\mathrm{X}$, Braun J. Imaging scoring methods in axial spondyloarthritis. Rheum Dis Clin North Am 2016;42:663678.

31. Rudwaleit M, Landewe R, van der Heijde D, et al. The development of Assessment of SpondyloArthritis International Society classification criteria for axial spondyloarthritis (part I): classification of paper patients by expert opinion including uncertainty appraisal. Ann Rheum Dis 2009;68:770-776.

32. Tuite MJ. Sacroiliac joint imaging. Semin Musculoskelet Radiol 2008;12:72-82.

33. de Winter J, de Hooge M, van de Sande M, et al. Magnetic resonance imaging of the sacroiliac joints indicating sacroiliitis according to the Assessment of SpondyloArthritis International Society definition in healthy individuals, runners, and women with postpartum back pain. Arthritis Rheumatol 2018;70:1042-1048.

34. Bae JM, Choo JY, Kim KJ, Park KS. Association of inflammatory bowel disease with ankylosing spondylitis and rheumatoid arthritis: a nationwide population-based study. Mod Rheumatol 2017;27:435-440.

35. van der Linden S, Valkenburg HA, Cats A. Evaluation of diagnostic criteria for ankylosing spondylitis: a proposal for modification of the New York criteria. Arthritis Rheum 1984;27:361-368.

36. Geijer M, Gadeholt Gothlin G, Gothlin JH. The validity of the New York radiological grading criteria in diagnosing sacroiliitis by computed tomography. Acta Radiol 2009;50:664-673.

37. Peeters H, Vander Cruyssen B, Mielants H, et al. Clinical and genetic factors associated with sacroiliitis in Crohn's disease. J Gastroenterol Hepatol 2008;23:132-137.

38. Hiller A, Biedermann L, Fournier N, et al. The appearance of joint manifestations in the Swiss inflammatory bowel disease cohort. PLoS One 2019;14:e0211554.

39. Molnar T, Tiszlavicz L, Gyulai C, Nagy F, Lonovics J. Clinical significance of granuloma in Crohn's disease. World J Gastroenterol 2005;11:3118-3121. 